Documentation et bibliothèques

DOCUMENTATION BIBLIOTHEQUES

\title{
Services documentaires et sciences de l'information
}

\section{Yves Courrier}

Volume 20, numéro 3, septembre 1974

URI : https://id.erudit.org/iderudit/1055669ar

DOI : https://doi.org/10.7202/1055669ar

Aller au sommaire du numéro

\section{Éditeur(s)}

Association pour l'avancement des sciences et des techniques de la documentation (ASTED)

\section{ISSN}

0315-2340 (imprimé)

2291-8949 (numérique)

Découvrir la revue

Citer cet article

Courrier, Y. (1974). Services documentaires et sciences de l'information. Documentation et bibliothèques, 20(3), 147-158.

https://doi.org/10.7202/1055669ar

\section{Résumé de l'article}

Le but de cet article est d'étudier les rapports entre la bibliothéconomie, la documentation et les sciences de l'information. L'auteur expose d'abord l'impact de certaines techniques modernes sur la bibliothéconomie, en particulier la reprographie et l'informatique. Puis un parallèle est fait entre la bibliothéconomie et la documentation où l'on y souligne les points communs. Enfin, les sciences de l'information sont étudiées de façon interdisciplinaire, en abordant le concept d'information dans différentes sciences exactes et humaines.
Tous droits réservés ( $)$ Association pour l'avancement des sciences et des techniques de la documentation (ASTED), 1974
Ce document est protégé par la loi sur le droit d'auteur. L'utilisation des services d'Érudit (y compris la reproduction) est assujettie à sa politique d'utilisation que vous pouvez consulter en ligne.

https://apropos.erudit.org/fr/usagers/politique-dutilisation/ 


\title{
Services documentaires et \\ sciences de l'information
}

\author{
Yves Courrier \\ École de bibliothéconomie \\ Université de Montréal
}

Le but de cet article est d'étudier les rapports entre la bibliothéconomie, la documentation et les sciences de l'information. L'auteur expose d'abord l'impact de certaines techniques modernes sur la bibliothéconomie, en particulier la reprographie et l'informatique. Puis un parallèle est fait entre la bibliothéconomie et la documentation où l'on y souligne les points communs. Enfin, les sciences de l'information sont étudiées de façon interdisciplinaire, en abordant le concept d'information dans différentes sciences exactes et humaines.

Parler des changements de la société est devenu un lieu commun. Des ouvrages de vulgarisation et des études spécialisées essaient d'en déterminer l'impact sur les individus, les groupes sociaux, les institutions'. Pourtant, si l'on suppose que le moteur de ces changements est la science et la technologie, ceux qui sont le plus concernés semblent parfois négliger d'en tirer les conséquences.

Si le progrès est dû aux innovations scientifiques et techniques, les organismes producteurs de ce savoir en sont les premiers affectés. Puisque l'on fait des découvertes, que l'on dépose des brevets, que l'on invente des procédés, il faut diffuser ces nouveautés. En somme, les multiples transformations de la société résultent, pour beaucoup, de développements intellectuels au point d'aboutir à un problème très grave, nommé et identifié depuis les années soixante: l'explosion de l'information. La multiplication de la documentation s'est accompagnée, de surcroît, d'une diversification des supports et des circuits de l'information. Face à de tels bouleversements, les bibliothèques sont-elles devenues des institutions dynamiques? Les bibliothécaires ontils su renouveler leur méthode? Serons-nous capables de suivre - et même de prévoir le changement?

Superficiellement, les bibliothécaires ont répondu à cette interrogation de façons différentes: soit par l'adoption enthousiaste de la technologie disponible la plus récente, l'informatique, soit par une constatation amère: l'information n'est plus entre leurs mains.

1. Alvin Toeffler, Le Choc du futur, Paris, Denoël, 1971; Pierre Piganiol, Maitriser le progrès, Paris, Gonthier/Laffont, 1968
L'adoption de l'ordinateur, souvent trop rapide, était d'ailleurs favorisée par une surenchère et un phénomène de mode auquel les informaticiens se sont bien gardés de s'opposer. Mais, fait plus important, les développements proprement technologiques de l'informatique ont été confondus avec des découvertes théoriques simultanées mais pas nécessairement liées: théorie de l'information, cybernétique, théorie des systèmes, bionique, recherche opérationnelle, etc. Cependant, l'adoption de l'informatique n'a pu empêcher un défi: les bibliothécaires ont constaté qu'ils n'avaient plus le monopole de l'emmagasinage et de la transmission de l'information. A côté des bibliothèques traditionnelles (scolaires, publiques, universitaires, spécialisées, etc.) on a vu naître des centres de média, des centres de documentation, des informathèques, etc. On comprend que cette perte de monopole ait parfois suscité des réactions vigoureuses. Car de plus, les bibliothécaires constataient souvent que, sous des noms nouveaux, bien des documentalistes se contentaient de réinventer des méthodes fort traditionnelles; ou pire, restaient handicapés par l'ignorance d'un savoir-faire éprouvé depuis longtemps.

Les apparences permettent de constater à l'égard des nouveautés soit une confiance immodérée, soit des critiques un peu envieuses. Les deux attitudes posent en fait une question bien simple: quels sont les rapports entre la bibliothéconomie, telle que nous la connaissons, et l'ensemble des disciplines liées à l'information? Or, si la question est simple, la réponse ne l'est pas: car ces disciplines, parfois groupées sous le terme générique de Sciences de l'information, forment un ensemble assez confus. Pour disposer de repères dans ce domaine, $A$. Rees a proposé, en relation avec l'enseignement de la bi- 
bliothéconomie, une distinction de fait ${ }^{2}$. Son enquête lui permet de déterminer trois étiquettes sous lesquelles on peut grouper les domaines envisagés: l'informatique, les techniques documentaires, les sciences de l'information. Ce groupement, valable dans la mesure où il part d'un état de fait, offre cependant un cadre insuffisant pour donner une idée exacte des transformations récentes de la bibliothéconomie. L'informatique, au sens étroit d'une technologie, a eu un impact certain sur la bibliothéconomie, impact qui devra être développé. Mais d'autres techniques l'ont aussi influencée et méritent que l'on s'y arrête: l'audio-visuel, les divers moyens de reproduction des documents écrits, les télécommunications.

Le deuxième domaine - parfois appelé documentation - recouvre l'ensemble des techniques inventées dans les vingt ou trente dernières années pour répondre à des besoins d'information plus urgents: l'ordinateur est l'un de ces moyens; il est loin d'être le seul ni même le plus important.

Bref, il s'agira d'exposer d'abord les impacts des développements technologiques sur la bibliothéconomie. Puis l'on esquissera un historique des techniques documentaires. Alors, on pourra montrer que bibliothéconomie et documentation sont beaucoup plus proches qu' on ne l'a cru jusqu'à présent: leur unité se fonde même sur des raisons plus profondes que celles qui sont généralement avancées.

Les sciences de l'information, au contraire, recouvrent un ensemble beaucoup plus vaste de connaissances. Elles sont loin d'être un domaine structuré et, en tant que recherche encore très hésitante, elles devraient être mises à part. En d'autres termes, la distinction de fait - proposée par A. Rees - issue du renouvellement de la bibliothéconomie devra aboutir, et c'est ce qu'il faudra montrer, à une distinction de droit entre bibliothéconomie et documentation d'une part et les sciences de l'information d'autre part.

Mais auparavant, qu'entend-on par bibliothéconomie?

II est certes difficile de trouver un consensus à ce sujet. Tout d'abord, l'image du bibliothécaire a changé. Autrefois érudit-bibliophile-conservateur, il est devenu l'agent au service d'une communauté (école, lycée ou

2. Alan Rees, "Influence de la technologie des ordinateurs sur la formation bibliothéconomique, " Bulletin de I'Unesco à l'intention des bibliothèques, vol. 23, no 1 (janvier-février 1969), 28-33. collège, université, municipalité, parlement, etc.). Pour réaliser cette fonction, il doit faire preuve de connaissances dans beaucoup de domaines: culture, édition, techniques de communication, administration, éducation. On commence aussi à se rendre compte qu'il a parfois besoin d'utiliser des sciences fondamentales: psychologie, sociologie, linguistique, statistique, etc. Également, il doit maîtriser un certain nombre de savoir-faire précis pour l'acquisition, le stockage, la recherche et la diffusion des documents dont il a la charge. Mais quels que soient le support et la nature de ces documents, quelle que soit la modernité des techniques qu'il utilise, l'identité propre du blibliothécaire et la singularité de son rôle tiennent au service documentaire d'une clientèle définie. La bibliothéconomie est un savoir professionnel, au même titre que la médecine ou le droit, et, comme ces derniers, elle exige une déontologie ${ }^{3}$. En tant que tel, ce savoir peut se fonder sur des sciences, mais il n'est pas une science: c'est un savoir-faire au service de l'homme et de la société.

En ce sens, certaines critiques d'usurpation que les bibliothécaires adressèrent aux documentalistes sont compréhensibles. Comme pour le bibliothécaire, le rôle du documentaliste est de satisfaire des besoins documentaires et il est au service d'une population bien définie (entreprise, centre de recherche, laboratoire).

Est-ce à dire que bibliothéconomie et documentation sont en tous points semblables: certes non! Nul ne nie que la bibliothéconomie trouve ses origines dans le rôle de conservation des documents. La documentation par contre est née de besoins d'informations urgents. L'hétérogénéité des origines est évidente; de surcroit, on verra que le temps est venu accentuer les oppositions. Pourtant, il faudra dépasser ces divergences. Non seulement, il sera possible de discerner un mouvement de convergence, mais il s'agira de montrer que ce ne peut être qu'au bénéfice des deux professions. Bien sûr, on risquera les critiques de ceux qui pensent que leur tâche est unique: la conservation de livres précieux, la restauration d'ouvrages rares exigent des méthodes et des attitudes propres au bibliothécaire. Inversement, la manipulation de l'information dans une entreprise exige des moyens de contrôle certainement moins lourds que dans une bibliothèque, ou encore des procédés de diffusion plus agressifs. Et l'on pourrait

3. Laurent Denis, Réflexions d'un bibliothécaire sur sa profession, Montréal, Université de Montréal, École de bibliothéconomie, 1970. 
aisément multiplier les exemples. C'est cependant la recherche d'une unité qui nous arrêtera, car elle concerne la formation professionnelle et le renouvellement de la bibliothéconomie et de la documentation.

\section{Bibliothèques et technologie}

L'impact de la technologie sur la bibliothéconomie a pris quatre aspects principaux: les techniques audio-visuelles, les techniques de reproduction, l'informatique et les télécommunications.

Exposer le développement de l'audio-visuel pourrait faire l'objet d'une communication séparée. Sans même vouloir toucher à l'aspect des communications de masse, on doit cependant mentionner que les nouveaux supports de l'information ont obligé les bibliothécaires à considérer que la documentation ne se trouvait pas uniquement sur du papier: transparents, diapositives, fiches, films, bandes magnétiques ou magnétoscopiques, etc. Les bibliothèques, et plus particulièrement les bibliothèques liées à des institutions d'enseignement, devenaient des centres multi-média. Ces transformations du matériel de base exigeaient à leur tour une évolution des méthodes: l'acquisition des documents se fait d'après d'autres sources, les techniques d'identification, de description se doivent d'être plus précises, plus détaillées, plus près du contenu. L'emmagasinage exige des outils plus diversifiés et des techniques plus souples. La recherche a fait naître des instruments spécialisés et l'utilisation ne peut se faire qu'avec la contribution de spécialistes. Inutile de développer plus amplement ce point: il est suffisamment bien traité dans une documentation spécialisée. ${ }^{4}$

De leur côté, de nouveaux moyens de reproduction rapide ont fait naître différentes sortes de problèmes, les uns liés à la microreproduction, les autres liés à la photocopie en masse. Pour ce qui est des premiers, on commence certes à voir dans les bibliothèques des lecteurs de microfilms. Certains bibliothécaires savent que pour obtenir des exemplaires épuisés de périodiques, la microcopie est leur meilleure chance, qu'il est moins coûteux d'obtenir des articles en réduction, etc. Mais l'important est de noter que cette technologie devrait changer complètement l'attitude et les décisions administratives des bibliothécaires à l'égard de l'acquisition, de l'emmagasinage et de la récupération des ouvrages autrefois imprimés. II ne devrait plus

4. Voir par exemple W.B. Hicks et A.M. Tillin, Developing Multi-Media Libraries, New York, Bowker, 1970. être exclu d'avoir sur microfilm les ouvrages rarement lus, les numéros de périodiques qui ont plus de cinq ou dix ans, même dans une bibliothèque universitaire.

Les questions que soulèvent les procédés rapides de reproduction sont sans doute présentes à tous les esprits: droit d'auteur, rentabilité de l'édition, avenir des formes traditionnelles de diffusion du savoir. Mais l'attitude des bibliothécaires face à ces questions devrait être clarifiée. II ne suffit pas d'installer quelques machines à photocopier, ici et là, entre les rayons de périodiques. II faudrait étudier dans quelle mesure l'acquisition, l'emmagasinage et la distribution - voire même la transmission, puisqu'il existe déjà des appareils à photocopier à distance - des documents pourraient en être révolutionnés. D'autre part, dans les débats juridiques du droit d'auteur et du copyright, il semble que l'on ait mal réalisé que les bibliothécaires sont vraiment les premiers accusés: aux États-Unis, c'est la Bibliothèque nationale de médecine qui est en cause, et en France, le service de documentation du Centre national de la recherche scientifique.

Le troisième impact technologique, probablement le plus connu, est l'informatique. C'est un sujet brûlant qui suscite encore l'enthousiasme ou le mépris. Devant l'animosité des débats, certains ont alors préféré adopter l'attitude wait and see. Cependant, si cette dernière attitude est justifiable humainement parlant, elle risque de provoquer un retard irrécupérable à la fois pour l'efficacité des services techniques et la qualité des services à l'usager. Face à l'explosion de l'information, il est temps que les bibliothécaires recueillent au moins suffisamment d'informations pour prendre des décisions sages.

On pourrait objecter que dans cette optique, l'enthousiasme du milieu des années soixante a provoqué des catastrophes, plus particulièrement en Amérique: la documentation de cette époque fait état de nombreux projets, avec beaucoup de verbes au futur! On s'est vite aperçu que l'ordinateur n'était pas la solution miracle, et les échecs, plus ou moins bien camouflés, $n$ 'en ont pas moins été douloureusement ressentis et ont servi d'arguments aux partisans des méthodes traditionnelles. A un enthousiasme immodéré succède un scepticisme acerbe. Peut-être sommes-nous témoins actuellement de la découverte d'un équilibre ${ }^{5}$ ? Car les débats, s'ils ont été éprouvants, n'ont pas été inutiles; et

5. Henriette Avram, "Library Automation: a balanced view». Library Resources and Technical Services, vol. 16 , no. 1 (Winter 1972), 11-18 
I'on a su tirer des leçons d'expériences malheureusement très coûteuses.

L'automatisation des bibliothèques coûte cher: les bibliothécaires ignoraient les exigences de l'informatique et les informaticiens sous-estimaient les besoins des bibliothèques. Les premiers ont découvert que l'ordinateur est un instrument très difficile à manier; il est difficile de lui faire rendre les services que l'on voudrait. Les informaticiens, quant à eux, ont appris que mettre un catalogue sur ordinateur n'est pas chose facile, que les règles d'intercalation des fiches sont plus complexes qu'un simple tri alphabétique, que la plupart des données doivent être emmagasinées en champ variable, que le temps réel est la condition sine qua non pour certains services, etc.

Les leçons que l'on a tirées tiennent compte de toutes ces difficultés: l'automatisation doit être soigneusement planifiée, en tenant compte de l'ensemble des services et, surtout, elle doit être faite en coopération. Les seules expériences vraiment convaincantes sont des tentatives collectives: OCLC pour les acquisitions et le catalogage, la distribution des bandes MARC. Un système de prêt automatisé dans une petite bibliothèque peut certes rendre quelques services (économie de personnel, statistiques de circulation) mais ce ne peut être qu'une réalisation marginale sans grande incidence sur la bibliothéconomie.

Si donc l'automatisation des bibliothèques doit se faire, c'est en coopération, et l'on commence à peine à réaliser les difficultés que cela entraîne: difficultés psychologiques, politiques, administratives et financières. Ainsi, la normalisation internationale du catalogage est devenue inéluctable. Mais il faut admettre que les solutions nouvelles apportent des difficultés imprévisibles. Par exemple, si une bibliothèque décide d'organiser un réseau centralisé d'acquisition et de catalogage, il faut non seulement prévoir le partage des responsabilités administratives et financières, mais aussi inventer des mécanismes pour que les derniers venus assument le fardeau des frais de recherche, évidemment très lourds, que le groupe initial a bien voulu porter. Sinon, il suffirait d'attendre que les autres se lancent!

La concertation est donc exigeante: mais c'est là le véritable impact de l'informatique sur la bibliothéconomie, celui qui permettra de faire face à l'explosion de l'information grâce à l'unification des procédures et des normes. ${ }^{6}$ Et c'est d'autant plus la voie à suivre

6. Rosario de Varennes, «Informatique 1971: principales tendances dans le domaine de l'automatisation des fonctions bibliothéconomiques", Bulletin de l'ACBLF, vol. 17, no 2 (juin 1971), 54-61. qu'un quatrième aspect de l'impact technologique vient s'y ajouter: la révolution des télécommunications.?

Les bibliothécaires ont été moins sensibles à ce dernier aspect. II risque pourtant - mais sans doute à plus longue échéance - d'être aussi important que les deux premiers: depuis l'amélioration des circuits téléphoniques jusqu'aux satellites, en passant par la télévision par câble et les micro-ondes, la transmission d'énormes quantités de données est aujourd'hui techniquement possible, sinon économiquement rentable pour les bibliothèques. En fait, la volonté politique de coopérer et l'imagination administrative font plus souvent défaut que les ressources pécuniaires. Le fonctionnement des bibliothèques doit être une entreprise collective, tout en sachant que collaboration ne veut pas dire système centralisé où toute l'initiative vient d'un organisme tout-puissant. ${ }^{\text {a }}$ La coopération ne signifie pas la négation des besoins locaux pas plus que l'écrasement des initiatives individuelles. ${ }^{8}$

Que ce soit les techniques audio-visuelles, les techniques de reproduction, l'informatique ou les télécommunications, on voit que la technologie devrait avoir des incidences très concrètes sur le travail des bibliothécaires: d'un point de vue administratif, rationalisation des tâches et établissement de structures de coopération; d'un point de vue technique, acquisition, description, emmagasinage et utilisation d'un matériel plus hétéroclite et difficile à traiter, et établissement des voies de communication. L'impact du progrès amène, ou inéluctablement devra amener, une transformation importante de la bibliothéconomie. II s'agira de montrer que ce renouvellement ne se limite pas aux considérations pratiques qui viennent d'être exposées.

\section{Bibliothéconomie et documentation}

Si l'impact de la technologie sur la bibliothéconomie est relativement récent (les années soixante), on peut dire au contraire que les techniques documentaires - et elles

7. H.C. Campbell, "Quelques incidences des communications par satellites sur les bibliothèques». Bulletin de l'Unesco à l'intention des bibliothèques, vol. 20, no 3 (mai-juin 1966), 139-144.

7a. Au chapitre de la coopération et de la transmission des données, mentionnons une expérience des plus intéressantes qui est en voie de réalisation, celle du télécatalogage en coopération des universités du Québec et de l'Ontario.

8. Yves Courrier, "Automatisation des bibliothèques et projet MARC: le concept de format de communication". Bulletin de l'ACBLF, vol. 18, no 2 (juin 1972), 97-106. 
portent bien leur nom - ont suivi de très près l'innovation technologique. Jesse $H$. Shera mentionne que l'American Documentation

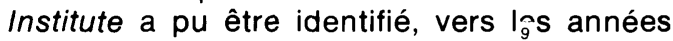
trente, avec la microphotographie." On pourrait aussi citer l'adoption des divers autres procédés de stockage (cartes à perforation marginale ou totale) et l'utilisation, dès les années cinquante, des cartes perforées, contemporaines des contributions très importantes de Mooers, Taube, Perry, Kent, Luhn, etc. ${ }^{10}$ Plus récemment encore, on constate l'exploitation des bandes magnétiques destinées aux photocomposeuses pour des services de diffusion sélective de l'information. Enfin, comme lieu privilégié de l'utilisation de la technologie, l'ordinateur se présente avec toutes ses possibilités: à toutes les étapes du traitement de la documentation, il apporte sa souplesse et ses capacités énormes. Certes, tous les problèmes des techniques documentaires ne sont pas résolus. Mais prendre conscience que de gros investissements sont nécessaires est souvent l'obstacle le plus important à franchir.

Ces différences d'attitude à l'égard de la technologie perçue, d'une part comme un impact plutôt reçu, d'autre part avec une attention soutenue aux développements, recouvrent une autre différence moins superficielle. Le documentaliste s'est toujours senti confronté à des besoins d'information urgents et précis. L'utilisation de moyens mécaniques, photographiques ou électroniques est une partie de la réponse. II faut y ajouter un point de vue différent: la documentation, plutôt que d'être emmagasinée et mise à la disposition de l'usager, doit être analysée et exploitée de façon active.

Un exemple pourrait éclairer cette différence. Depuis que les bibliothèques existent, on classe les ouvrages. L'introduction du libre accès est venu renouveler les problèmes de classification et les différents systèmes ont beaucoup évolué. Leur principe, cependant, à l'exception peut-être des classifications à facettes et de certaines classifications spécialisées, est de grouper les ouvrages en fonction d'une caractéristique commune afin de réunir ceux qui concernent le même sujet. Dans ce but, les classifications hiérarchiques sont l'outil idéal. Si on y a ajouté les vedettes-ma-

9. Jesse H. Shera, "Bibliothéconomie, documentation et science de l'information", Bulletin de l'Unesco à l'intention des bibliothèques, vol. 19 , no 1 (mars-avril 1968), $62-70$.

10. Jacques Chaumier, Les techniques documentaires, Paris, Presses Universitaires de France, 1971, p. 5-11; Gerard Salton, Automatic Information Organization and Retrieval, New York, McGraw-Hill, 1968. tière, c'est pour essayer d'introduire un peu de précision. A ce groupement des ouvrages, le documentaliste veut ajouter une autre dimension: faire ressortir les multiples concepts ou aspects des documents en les indexant, en les signalant. Au-delà du genre, de la caractéristique commune qui permet de grouper, il s'agit de trouver les différences spécifiques, à tel point que le documentaliste ignorera souvent le genre et s'abstiendra bien souvent de grouper les documents par sujets. Alors qu'une bibliothèque exige l'utilisation de classifications encyclopédiques et bien souvent hiérarchiques, un centre de documentation a besoin de langages d'indexation spécialisés: classification à facettes, thésauri, etc. Distinguer indexation précoordonnée et postcoordonnée n'est pas seulement un exercice scolaire $^{11}$. En multipliant les accès possibles aux documents, on prépare un repérage rapide et efficace. Le documentaliste attribue ainsi à l'information une valeur monétaire chiffrable. Le service de documentation devient une entreprise rentable. Les documentalistes ont peut-être senti plus rapidement que l'explosion de l'information est devenue un problème économique.

Ainsi, par suite de leurs origines diverses, bibliothéconomie et documentation ont subi, chacune à sa façon, l'impact de la technologie. A cela s'ajoute une opposition assez accentuée dans les attitudes. Cette opposition s'est résorbée ultérieurement dans une convergence de plus en plus marquée due à l'identité des problèmes (explosion de l'information) et des méthodes (nécessité d'utiliser des moyens techniques). On comprend pourquoi les liens et les contacts entre les deux professions se renforcent et se multiplient. ${ }^{12}$ II ne s'agit pas d'une tentative de récupération de la part de l'une ou de l'autre. II pourrait s'agir, bien au contraire, d'un mouvement profond qui correspond sans doute à une meilleure compréhension, par chacune des professions, de leur nature propre. On a vu plus haut que bibliothéconomie et documentation étaient, toutes deux, des professions de service pour une population déterminée. On pourrait certes différencier des publics respectifs: d'un côté un public assez vaste, "encyclopédique", avec des intérêts culturels ou scientifiques où le temps n'est pas toujours

11. F Lancaster, Vocabulary Control for Information Retrieval, Washington, Information Resources Press, 1972.

12. Ainsi l'Association canadienne des bibliothécaires de langue française (ACBLF) a modifié sa raison sociale pour Association pour l'avancement des sciences et des techniques de la documentation (ASTED) après avoir changé le titre de son Bulletin pour Documentation et bibliothèques. 
un élément primordial; de l'autre côté, une clientèle restreinte, hautement spécialisée, pour qui la rapidité et la valeur économique de l'information sont cruciales. Mais ces différences sont exagérées. II serait facile de montrer qu'il y a toute une gamme de services documentaires entre ces deux extrêmes; le plus important n'est pas la typologie des publics, mais la nature du service rendu. Dans les deux cas, il s'agit d'un service documentaire dont le but est de procurer à une personne donnée, au moment voulu, une information pertinente. Bibliothécaires et documentalistes manipulent l'information enregistrée - quel qu'en soit le support - pour qu'elle soit utilisée.

Alors, puisque le service est le même, on comprend que les fonctions soient communes: collecte, analyse, emmagasinage, exploitation et diffusion de l'information. Collecte des documents et service des acquisitions ne doivent-ils pas répondre aux mêmes exigences: capacité de choisir dans une masse toujours croissante de documents, rapidité du service et adéquation à une clientèle toujours plus exigeante? L'analyse documentaire (ce que les bibliothécaires appellent catalogage) ne consiste-t-elle pas à décrire les éléments d'origine et le contenu d'un document ${ }^{13}$ ? Les différences d'attitude au niveau de l'analyse ont été soulignées par l'exemple de la classification. Elles se résorbent actuellement car les bibliothécaires doivent être capables de décrire de plus en plus précisément leurs documents, alors que les documentalistes pourraient tirer profit de certains principes généraux découverts à l'occasion de la constitution de classifications encyclopédiques. On pourrait même ajouter que, s'il est vrai que l'analyse des documents se fera de plus en plus dans des grands centres ${ }^{14}$, la nécessité de vastes systèmes de classification va à nouveau se faire sentir. Pour ce qui est de l'emmagasinage, il s'agit évidemment de l'impact des techniques de reproduction et de l'informatique, mentionné plus haut, où l'expérience acquise par les documentalistes pourrait être exploitée.

Enfin, au niveau de l'exploitation et de la diffusion, on pourrait montrer comment la référence et la recherche documentaire sont, finalement, deux fonctions très proches. Dans les deux cas, il s'agit d'identifier des besoins

13. "Analysis" in Encyclopedia of Library and Information Science, Volume I, publié sous la direction de A. Kent et H. Lancour, New York, Marcel Dekker, 1968.

14. "Les Tendances actuelles de la documentation automatisée», in J. Péguet et G. Gasty, éd., Développements actuels de l'informatique de gestion, Paris, Entreprise Moderne d'Édition, 1970, p. 209-222. et d'utiliser des outils et des techniques de recherche ayant des principes identiques ${ }^{15}$.

On pourrait évoquer mille autres possibilités d'enrichissement réciproque. G. Salton propose de nouvelles formes de manipulation et d'exploitation de fichiers de bibliothèque sur la base de l'expérience acquise en informatique documentaire ${ }^{16}$. La notion de pertinence (anglais: relevance) pourrait également être utilisée avec fruit dans tous les cas où il y a service documentaire. Fonctions identiques, intégrées pour le même service et emploi d'une technologie très apparentée, la recherche d'une unité ne devrait plus être à faire. Les conséquences de cette unité devraient plutôt être le véritable objet des préoccupations actuelles. Comme il a déjà été suggéré, l'échange d'idées devrait apporter des améliorations dans les pratiques professionnelles respectives et suggérer des domaines d'investigation expérimentale. On pourrait aussi envisager, pour la formation des bibliothécaires et des documentalistes, un noyau commun assez important, même si l'utilisation de techniques particulières exige une spécialisation.

\section{Les sciences de l'information}

Au-delà de ce rapprochement, identifiable par la communauté de services et de fonctions, n'est-il pas possible de chercher un savoir plus fondamental? Bibliothécaires et documentalistes sont concernés par l'information. Un certain nombre de connaissances sur ce sujet sont acquises. L'information, nous le savons, est une entité multiforme. C'est un phénomène individuel et collectif que certains mesurent ou traitent; elle circule et elle est enregistrée. Elle est utilisée, absorbée, répandue, diffusée, manipulée, analysée, synthétisée. Cas rare dans l'économie: on peut la distribuer sans qu'elle perde de sa valeur c'est même souvent le contraire qui se produit. Et pourtant, il est extrêmement difficile de contrôler ou de chiffrer sa productivité ou son utilité. Les multiples facettes de l'information justifieraient ainsi un savoir interdisciplinaire et la contribution d'un grand nombre de disciplines: sciences pures, exactes ou humaines, (mathématique, physique, chimie, biologie, psychologie, sociologie, linguistique) sciences appliquées (cybernétique, statistique, recherche opérationnelle, informatique, théorie de l'information, économie,

15. Robert S. Taylor, "Question-Negotiation and Information Seeking in Libraries", College and Research Libraries, vol. 29, no. 3 (May 1968), 178-194.

16. Gérard Salton, "Proposals for a Dynamic Library", Second Revision, Information. Part 2 Reports - Bibliographies, vol. 2, no. 3 (May 1973), 1-27. 
politique) savoirs professionnels (bibliothéconomie, documentation, analyse des systèmes, management, administration). L'important n'est pas tellement l'attribution dans les catégories qui pourrait être remaniée, mais plutôt le fait que tous ces exemples sont réels et justifiables. Chacun de ces points de vue est un apport potentiel, et seulement potentiel, à une étude de l'information. Car il faut bien l'avouer, trop souvent, sous couvert d'interdisciplinarité, les sciences de l'information ne sont qu'un fourre-tout. On ne sait ni ce qu'elles sont, ni ce que l'on veut en faire, à tel point que certains les ont définies "une maison bâtie sur le sable» ${ }^{17}$.

Cet état de choses aurait des incidences positives si la diversité des points de vue permettait des découvertes fondamentales. II semble que ce ne soit pas le cas: on assiste plutôt à une logomachie sur les définitions, les domaines pertinents, les méthodes de recherche, etc. Un développement sensé de la recherche et de l'enseignement nécessite des catégories plus claires, sinon plus rigides.

Bien sûr, c'est un phénomène courant dans l'histoire des sciences qu'un domaine se définisse lentement, qu'il cherche son objet propre et qu'il ne se structure que petit à petit. Fréquemment, des sciences exactes sont nées de savoirs professionnels: il y a eu des arpenteurs avant Euclide, des ingénieurs avant les physiciens, des médecins avant les biologistes et des apothicaires avant les chimistes. L'analogie mériterait une étude plus approfondie, mais il ne serait pas irraisonnable de postuler que si une science de l'information doit se créer, c'est probablement en se détachant des problèmes strictement professionnels tels qu'ils ont été décrits plus haut.

On pourrait aussi examiner la situation à l'aide des descriptions que T. Kuhn a données pour le développement des sciences exactes et qu'il croit valables également pour les sciences humaines ${ }^{18}$. Une science mûre se reconnaît à son "paradigme», c'est-à-dire un corpus de connaissances et de méthodes, un consensus sur les résultats, les faits pertinents, l'objet même de cette science. L'ensemble peut se trouver explicitement dans des manuels ou rester implicite et transmis par des pratiques professionnelles ou de laboratoire. On pourrait aussi mentionner les sociétés sa-

17. Louis Vagianos, «Information Science: a house built on sand", Library Journal, vol. 97, no. 2 (January $15,1972), 153-157$

18. Thomas S. Kuhn, The Structure of Scientific Revolutions, Chicago, The University of Chicago Press, 1970. La traduction française de ce volume a paru chez Flammarion sous le titre La structure des révolutions scientifiques, 1972 vantes et les publications spécialisées comme signes manifestes de ce paradigme. Actuellement, il est clair que les sciences de l'information, non seulement n'exhibent que très peu des caractéristiques d'une science qui a trouvé son paradigme, mais encore peuvent se donner très aisément les attributs d'une science à un stage préparadigmatique: collecte presque aléatoire des faits, multiplication de vues divergentes sinon contradictoires sur l'objet même des sciences de l'information, sur les théories possibles, les méthodes à envisager, les données à collecter, les phénomènes à observer. Et s'il existe quelques revues spécialisées, le lecteur profane verra rapidement que le contenu et la qualité des articles sont très inégaux; quant au chercheur, il sait très bien que ces revues couvrent très mal ses besoins. Encore une fois, quel que soit le point de vue épistémologique adopté, il est clair qu'il n'existe actuellement aucune entente sur ce qui fait l'unité des sciences de l'information.

II ne saurait être question de présenter, dans les lignes qui suivent, une structuration des sciences de l'information qui n'existe pas encore. Pourtant, il faut bien présenter quelques tendances qui se dessinent de plus en plus précisément et qui illustrent notre propos: la recherche en sciences de l'information s'éloigne des réalités professionnelles du bibliothécaire et du documentaliste. La situation actuelle suggère d'ailleurs deux approches pour une première investigation. D'une part, puisque le point commun semble être l'information, pourquoi ne pas jeter un coup d'œil sur quelques tentatives de définition? Même s'il apparaît que sous le même terme, des phénomènes bien disparates sont mentionnés, au moins aura-t-on précisé la terminologie et les différences. D'autre part, puisque autour de ce point commun gravite un grand nombre de disciplines, pourquoi ne pas essayer d'aborder les sciences de l'information par ce qui semble être une de leurs caractéristiques les plus saillantes, une des rares qui obtiennent un consensus quasi-total: l'interdisciplinarité? Cette recherche de définitions et de méthodes permettra, de surcroît, d'évoquer quelques progrès notables des sciences de l'information.

L'étude du premier point - tentatives pour cerner la nature même de l'information - est loin d'être facile, car les définitions que l'on trouve relèvent de disciplines absolument différentes: mathématique, physique, biologie. Comment le même mot - information - peut-il référer à la même réalité?

Du fait que l'information peut être codée, certains l'ont réduite à une unité élémentaire 
tout à fait abstraite. C'est la thèse que défendent les partisans de l'application ou de la théorie mathématique de la communication (Shannon-Weaver) à tous les phénomènes qui concernent l'information ${ }^{19}$. L'avantage évident de cette attitude est qu'elle fournit une mesure précise. L'information est quantifiée en termes de probabilités: plus un événement ou son symbole est probable, moins son occurrence apporte d'information. On ne saurait nier les bénéfices que l'on peut tirer d'une telle théorie non seulement dans les applications concrètes de manipulation de l'information codée (transmission), mais aussi dans des domaines tels que la psychologie ou la sociologie. Est-ce à dire que la théorie mathématique donne une description adéquate de la nature du phénomène information? On conçoit que la théorie mathématique s'applique précisément aux problèmes de codage: il s'agit d'un objet physique où toutes les variables du système de transmission, c'est-à-dire tous les signes possibles, sont connues, même si leurs probabilités respectives sont seulement estimées. II est facile dans ces conditions d'attribuer une valeur numérique précise à un signal. II n'est pas sûr que le modèle soit aussi strictement valable lorsqu'on étudie des phénomènes où l'information est transmise de personne à personne, entre individus humains, et le plus souvent en utilisant le langage. Dans la plupart des cas, la totalité des événements possibles n'est pas connue. II ne s'agit pas de nier les apports possibles d'un modèle mathématique; on veut simplement souligner qu'il n'indique rien sur la nature même de l'information. La théorie mathématique de l'information a sans doute le même statut que les statistiques. On peut l'appliquer à bien des domaines différents, mais cette application ne peut pas servir d'argument quant à la nature de l'objet étudié. Lorsqu'on utilise la théorie de Shannon Weaver, à l'extérieur du cadre dans lequel elle a été inventée, c'est en tant que modèle, sans plus. On verra cependant plus loin que le statut de modèle pourra être précisé.

Au-delà de la théorie purement mathématique, d'autres ont voulu définir l'information par rapport à des phénomènes physiques, en relation avec des concepts fondamentaux tels qu'énergie ou entropie. II s'agit d'ailleurs d'une conséquence de la première attitude, car beaucoup, constatant le parallélisme formel, ont voulu voir un seul et même phénomène dans la mesure mathématique de l'information et la "néguentropie», ou la possibilité, en introduisant de l'«ordre», c'est-à-

19. Par exemple Abraham Moles, Roger Meetham, Jack Belzer, B. Mandelbrot.

20. Par exemple L. Brillouin, D. Mackay dire de l'information, d'échapper à la loi fondamentale des phénomènes physiques qui est l'accroissement de désordre (ou entropie) ${ }^{20}$. La tendance vers un état statistiquement plus probable mais moins ordonné, loi inéluctable du monde physique, serait contrebalancée par l'introduction d'information ou d'ordre. II existe déjà des tentatives où énergie (au sens physique) et information (au sens strict de la théorie mathématique) sont mises directement en rapport dans le contexte d'une utilisation humaine de l'information ${ }^{21}$. II faut malheureusement signaler que l'accord est loin d'être fait parmi les physiciens sur ces idées; même si le consensus n'est pas la condition nécessaire pour qu'une vérité soit reconnue comme étant d'ordre scientifique, les divergences sont encore telles qu'il serait présomptueux, pour les non-spécialistes, de considérer comme acquise une définition de l'information en termes purement physiques.

En effet, les processus biologiques semblent capables de se soustraire au deuxième principe de la thermodynamique, à savoir l'accroissement nécessaire du désordre dans un système fermé. Un être biologique peut non seulement maintenir sa structure, mais il peut la reproduire et la multiplier. II devient alors possible de suggérer que ces caractéristiques sont dues à une fonction créatrice d'ordre qui serait précisément l'information:

«... toute acquisition d'information suppose une interaction par elle-même consommatrice d'énergie [...] Ce théorème nous intéresse ici en ce que les enzymes exercent précisément, à l'échelle microscopique, une fonction créatrice d'ordre»" ${ }^{22}$.

On pourrait alors remonter l'échelle de complexité des êtres. Ainsi, il est intéressant de noter que les récepteurs, à l'intérieur des organes sensoriels, ne sont que des mécanismes qui transforment l'énergie du monde extérieur en signaux utilisables par l'organis$\mathrm{me}^{23}$. De là, la perception peut être abordée comme une étape d'intégration des phénomè-

21. M. Tribas et $E$. Mclrvine, "Energy and Information», Scientific American, vol. 225, no. 3 (September 1971), 179-184, 186, 188

22. Jacques Monod, Le hasard et la nécessité, Paris, Seuil, 1970, p. 73. Le débat, ici comme en physique, semble loin d'être clos. Voir: Prirogine, Illya., "La Thermodynamique de la vie". La Recherche, no. 24 (juin 1972), 547-562 et Schoffeniels, E., L'anti-hasard, Paris, Gauthier-Villars, 1973.

23. Pour une optique interdisciplinaire (théorie de l'information, informatique, cybernétique, neurophysiologie, linguistique) voir: Encyclopaedia of Linguistics, Information and Control, Edited by A.R. Meetham, Oxford, Pergamon Press, 1969. 
nes sensoriels, sans doute rendue possible par une capacité d'abstraction. Piaget a suffisamment bien démontré la continuité entre les phénomènes biologiques et l'intelligence humaine, cette dernière n'étant que l'organe spécialisé et différencié de l'interaction de l'individu et de l'environnement, l'instrument de l'adaptation ${ }^{24}$.

II devient tentant alors d'étudier comment l'être humain peut passer de l'abstraction à la symbolisation, et de la symbolisation au langage, par l'intermédiaire de la notion de signification ${ }^{25}$. La linguistique, aidée de la psychophysiologie et de l'informatique, devient ainsi un instrument privilégié pour l'étude de l'information. Ajoutons une dernière touche au tableau et mentionnons comment l'information utilisée par les groupes sociaux, les institutions ou les individus est liée à la décision $^{26}$. Cette façon de présenter les différents points de vue par lesquels on peut aborder l'information est trop facile pour être enrichissante. La recherche sur la nature de l'information n'en est guère plus avancée; même si ce point de vue peut être un essai d'organisation, il risque aussi d'être une tentative de réduction.

L'histoire des sciences, là encore, incite à la prudence. On a cru pouvoir réduire tous les phénomènes biologiques à des réactions physicochimiques élémentaires ${ }^{27}$. Pourtant, ce n'est pas parce que l'on connaît les mécanismes fondamentaux nécessaires à la vie que l'étude scientifique des phénomènes biologiques $n$ 'a besoin que des concepts des sciences plus élémentaires. En d'autres termes, un biologiste, pour décrire ou expliquer les phénomènes qu'il étudie, a besoin de concepts tels qu'évolution, téléonomie, cellule et biologie. Reconnaître que «la phénoménale complexité des systèmes vivants [...] défie toute représentation intuitive globale ${ }^{28}$ est plutôt un sophisme. Des niveaux différents de com-

24. Jean Piaget, Biologie et connaissance, Paris, Gallimard, 1967

25. Jean Piaget, Le langage et la pensée chez l'enfant, Neuchâtel, Delachaux, 1924. Pour des travaux plus récents, Pierre Oléron, Langage et développement mental, Bruxelles, Dessart, 1972.

26. Voir par exemple M. Yovits, "Information Science toward the development of a true scientific discipline", American Documentation, vol. 20, no. 4 (October 1969), 369-376, ou A. Etzioni, The Active Society. New York, Free Press, 1968

27. Si J. Monod est convaincant en rejetant l'anthropocentrisme, il l'est moins en montrant que, puisque la biosphère serait compatible avec une théorie générale de l'univers, elle n'en serait qu'un événement particulier

28. J. Monod, Le hasard et la nécessité, p. 156 plexité exigent des concepts, des théories et des explications différentes. Le réductionnisme ${ }^{29}$, déjà invitant dans des domaines jouissant d'une longue histoire, devient un véritable danger pour les sciences de l'information. Certains concepts des sciences exactes (information, entropie, codage) leur ont parfois été utiles; ils ne doivent en aucun cas les mener au réductionnisme.

Tout ceci risque de montrer une image peu séduisante des sciences de l'information. Présentées comme confuses, préparadigmatiques, réductionnistes même, comment peuton les considérer? Pourquoi prétendre qu'elles devraient se détacher nettement de la bibliothéconomie et de la documentation, si leur existence même peut être mise en question? Où chercher un fondement réel au noyau commun des deux professions qui nous ont semblé si proches?

Au lieu de plaquer, peut-être un peu trop rapidement, des concepts des sciences exactes, il serait prudent d'examiner de plus près les phénomènes auxquels on se réfère lorsqu'on parle d'information. Si l'on veut bien accepter ce minimum de discipline intellectuelle, on s'apercevra que les sciences de l'information ne se réduisent pas à la théorie de l'information, ni à aucune des sciences exactes que nous avons mentionnées.

II est en effet difficile de nier - y compris pour bien des cas de manipulation informatique de l'information - qu'il s'agit d'information non numérique. Et même lorsque c'est le cas, l'information n'est information que parce qu'elle est utilisée. Bien qu'elle se réduise parfois à des données, l'information est bien plus fréquemment une intégration de ces données; sans aller jusqu'à considérer l'information comme un savoir, il faut reconnaître qu'elle est, le plus souvent, sous une forme symbolique qui nécessite ou appelle une interprétation: langage humain ou tout autre système de symboles. En d'autres termes, l'information est générée par des êtres humains, pour des êtres humains, dans un cadre humain.

Cela peut paraître trivial, mais on néglige

29. Pour une approche récente, scientifique plutôt que philosophique et interdisciplinaire des problèmes du réductionnisme, voir: Beyond Reductionism, Boston, Beacon Press, 1971

Aucun des grands dictionnaires de langue ou de philosophie ne mentionne "réductionnisme". c'est-àdire la tendance philosophique qui cherche à expliquer le supérieur par l'inférieur. Le terme est cependant employé par D. Dubarle et J. Piaget. J. Monod et J. Piaget se servent aussi de l'adjectif "réductionniste». 
trop souvent que l'information naît d'une symbolisation proprement humaine, le langage. II est reconnu que les capacités verbales des primates sont très limitées; ce qu'on appelle les langages animaux sont, en fait, des systèmes sommaires de symbolisation ${ }^{30}$. Le cadre dans lequel l'information circule ou est utilisée est aussi proprement humain: les circuits de l'information et les groupes sociaux liés par eux. L'homme politique ou le journaliste, le chef d'entreprise ou le chercheur scientifique, l'écrivain ou l'étudiant, en fait tout être humain dans sa vie professionnelle ou privée, sont les producteurs et les utilisateurs de l'information. De même, l'information ne circule que par l'intermédiaire de groupes sociaux déterminés; qu'il s'agisse de couvrir l'événement, la culture ou le savoir scientifique, la transmission de l'information est assurée par les moyens les plus divers: moyens de communication de masse, mais aussi maisons d'édition, bibliothèques, sociétés scientifiques, institutions d'enseignement.

Tout ceci n'est pas très original et correspond à une définition, déjà ancienne et souvent citée, de la science de l'information:

"La science de l'information est cette discipline qui étudie les propriétés et le comportement de l'information, les forces qui en gouvernent le flot, et les moyens de la traiter pour une accessibilité et un usage optimum. Elle s'occupe de ce corps de connaissances lié à la création, la collecte, l'organisation, l'emmagasinage, le repérage, l'interprétation, la transmission, la transformation et l'utilisation de l'information ${ }^{31}$.

On pourrait objecter que, par cette définition, on risque une fusion avec les sciences de la communication. Ce risque n'est pas à exclure a priori; une telle fusion ne ferait que confirmer ce dont il est question ici. S'il existe une science de l'information, c'est une science humaine:

"La science de l'information semble avoir émergé non seulement comme une expansion et une métamorphose de la documentation et de la recherche documentaire: elle

30. Voir les travaux classiques de Lorenz et Gardner. Un résumé récent: E.P. Wilson, "Animal Communication", Scientific American, vol. 227, no. 3 (September 1972), 53-60, 66-69.

31. H. Borko, "Information Science: What is it?", American Documentation, vol. 19, no. 1 (January 1968), 3. La traduction, ainsi que celles qui suivent, sont nôtres. G. Harmon (voir note 32) cite une définition très proche, plus ancienne (1966), attribuée à C.A. Cuadra. incorpore directement ou indirectement plusieurs objectifs et concepts des sciences de la communication, du comportement et d'autres disciplines contributives. Elle leur est parallèle [...] La science de l'information, ainsi que la documentation et la recherche documentaire, s'est identifiée aux disciplines des communications. Les tentatives récentes pour définir la science de l'information suggèrent un lien avec la génération moderne des sciences de la communication et du comportement ${ }^{32}$.

Peut-être avons-nous déjà trop développé cet aspect des sciences de l'information. Et pourtant, $y$ insister oblige à examiner attentivement certaines caractéristiques propres aux sciences humaines. Piaget a démontré que les sciences humaines se différencient des sciences exactes sur deux points essentiels ${ }^{33}$ : l'absence d'un ordre hiérarchique lié à la filiation des notions et l'absence du réductionnisme. En essayant de préciser le statut des sciences de l'information, il s'agit donc de se garder du réductionnisme, puisqu'aucun critère ne permet d'organiser les différentes sciences, et surtout d'accepter l'interdisciplinarité comme état normal. Souligner l'interdisciplinarité des sciences de l'information n'est pas chose nouvelle non plus. Mais il faut, là aussi, se refuser à la confusion ou à la facilité. Et ceci n'est guère aisé, car l'interdisciplinarité, sans être une approche d'invention récente, a été étudiée en tant que telle depuis peu seulement ${ }^{34}$. On commence à peine à distinguer ses différentes formes et pourtant une étude sérieuse permettrait bien des éclaircissements.

Une des formes les plus anciennes et les plus connues d'interdisciplinarité est probablement celle où une discipline est obligée de faire appel à d'autres domaines qui lui sont marginaux. Très courants dans les sciences exactes (biochimie, chimie, physique, etc.), les exemples dans les sciences humaines sont plus contestables: la psychologie sociale n'est-elle pas très difficilement identifiable soit à la psychologie, soit à la sociologie? C'est probablement aussi le cas pour les sciences

32. Glynn Harmon, "On the Evolution of Information Science". Journal of the American Society for Information Science, vol. 22, no. 4 (July-August 1971), 240.

33. Jean Piaget, Épistémologie des sciences de l'homme, Paris, Gallimard, 1970, p. 254-256.

34. L'interdisciplinarité. Problèmes d'enseignement et de recherche dans les universités, OCDE, CERI, 1972.

Tendances principales de la recherche dans les sciences sociales et humaines. Première Partie: Sciences Sociales, Paris, Mouton/Unesco, 1970.

Nous utiliserons surtout la synthèse proposée par F. Russo, "La pluridisciplinarité", Études, vol. 338, no 5 (mai 1973), 763-780. 
de l'information. Domaine encore mal structuré, les rapports avec des domaines connexes sont aussi très difficilement identifiables ${ }^{35}$.

Une autre forme d'interdisciplinarité, parfois nommée transdisciplinarité, pourrait être plus éclairante. II s'agit du cas où

«au sein de plusieurs disciplines, soit voisines, soit fort différentes, [...] une discipline de caractère général [...] procure une intelligence plus profonde et plus exacte des objets, des processus, des phénomènes que visent ces disciplines ${ }^{36}$.

A ce sujet, F. Russo cite, entre autres, la théorie de l'information et la sémiologie. Envisager un tel rapport antre la théorie de l'information ou la sémiologie d'une part, et les sciences de l'information d'autre part est tentant, car cela mènerait à des conséquences intéressantes. En effet, ce point de vue s'accompagne d'exigences assez strictes. II obligerait d'abord à admettre la spécificité des domaines, ce qui permettrait de se garder du réductionnisme mentionné plus haut. Ensuite, l'application de la "transdiscipline» ne serait fructueuse que si elle ne se faisait qu'avec extrêmement de rigueur et de précision. Ceci devrait mener à une recherche de méthodes qui satisfasse cet aspect. Enfin, il faudrait que le modèle soit cohérent et qu'il apporte des vues vraiment nouvelles aux disciplines particulières. Ce serait l'aspect le moins contestable de l'introduction de la théorie de l'information aux sciences de l'information, alors que les deux premières exigences semblent avoir fait défaut bien trop souvent.

Plus rigoureusement sans doute, J.C. Gardin a montré, bien qu'il n'emploie pas le terme, comment une sémiologie pourrait être la transdiscipline de quatre domaines assez différents: l'analyse documentaire, l'anthropologie structurale, l'analyse du contenu en sociologie et l'analyse littéraire ${ }^{37}$. Ces disciplines utiliseraient pour des problèmes semblables - l'élucidation d'une signification cachée - une démarche commune dont le modèle et les conditions de validation sont explicités.

35. Peut-être pourrions-nous envisager de ce point de vue la relation entre les sciences de l'information et de la communication que nous évoquions plus haut. Malgré les dangers de confusion et de superficialité que nous dénoncions plus haut, on est obligé d'admettre que ces deux domaines sont encore trop neufs pour être structurés et pour formaliser leurs liens réciproques.

36. François Russo, "La pluridisciplinarité», 770.

37. Jean-Claude Gardin, "Semantic Analysis Procedures in the Sciences of Man". Social Sciences Information, vol. 8, no. 1 (February 1969), 17-42.
Même si cette voie commence seulement à être explorée, elle n'en fournit pas moins de forts arguments pour une approche humaine et interdisciplinaire de certains aspects des sciences de l'information.

Les modes de l'interdisciplinarité ne sont pourtant pas épuisés. II reste celui où

«[...] les rapports entre les disciplines sont plus que des rapports de simple articulation, d'échange d'informations, de fourniture d'instruments ou de subordination, mais où plusieurs disciplines s'associent dans une coopération étroite pour l'élucidation d'un thème, l'intelligence d'un processus, la compréhension d'un ensemble de phénomènes» ${ }^{38}$.

Cette attitude pourrait, semble-t-il, caractériser les travaux où analyse documentaire et linguistique sont étroitement associées ${ }^{39}$. Le problème linguistique des universaux, qui a d'ailleurs été pendant fort longtemps un problème philosophique, pourrait bien rejoindre l'analyse documentaire. Cette dernière exige que les concepts des sciences particulières et leurs relations deviennent manipulables. La linguistique, quant à elle, propose l'élucidation des relations syntagmatiques et paradigmatiques des unités du langage. On peut alors entrevoir une interdisciplinarité profonde entre linguistique et analyse documentaire, interdisciplinarité qui s'orienterait vers l'analyse des concepts spécifiques aux sciences et à leurs relations. L'apport de la linguistique serait très valable au plan des méthodes de description éprouvées depuis beaucoup de temps déjà; l'apport des techniques d'analyse documentaire consisterait dans les connaissances déjà acquises empiriquement au niveau du contrôle du vocabulaire et de d'autres aspects de l'analyse documentaire. On rejoindrait peut-être, par un autre biais et de façon plus rigoureuse, les tentatives philosophiques d'analyse componentielle du

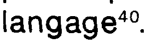

Un mode ancien d'interdisciplinarité, représenté de façon attirante en sciences de

38. François Russo, "La pluridisciplinarité", 773.

39. Voir par exemple Maurice Coyaud, Linguistique et documentation, Paris, Larousse, 1972; C.A. Montgomery, "Linguistics and Information Science», Journal of the American Society for Information Science, vol. 23, no. 3 (May-June 1972), 195-219. Jean-Claude Gardin, "Document Analysis and Linguistic Theory", The Journal of Documentation, vol. 29,no. 2 (June 1973), 137-168

40. R. Carnap, Y. Bar-Hillel, entre autres. Voir New Horizons in Linguistics, édité par J. Lyons, Penguin Books, 1971, p. 166-184 
l'information ${ }^{41}$, permettra de clore ce tour d'horizon. II s'agit de ce que F. Russo appelle la «pluridisciplinarité de synthèse»:

"recherche d'un principe qui rassemblerait [les savoirs] dans une vue globale où chacun serait exactement situé et où seraient clairement aperçues des relations avec les autres ${ }^{42}$.

Cette forme d'interdisciplinarité serait peutêtre la meilleure façon de caractériser les travaux de Churchman et de ses épigones Ackoff, Mitroff, etc. ${ }^{43}$ On pourrait croire que l'on retrouve simplement le cas précédent, c'est-à-dire un lien étroit entre deux disciplines, l'épistémologie et les sciences de l'information. En fait, le projet est beaucoup plus ambitieux: les différentes écoles épistémologiques (empirisme, rationalisme, rationalisme critique, hégélianisme) représenteraient différentes méthodes pour recueillir, organiser et utiliser l'information. En devenant des outils précis de manipulation de l'information, les systèmes philosophiques apportent en même temps, aux sciences de l'information, leur propre capacité de synthèse ${ }^{44}$.

\section{Conclusion}

Notre exploration pourrait continuer longtemps encore parmi les dédales des sciences de l'information. L'histoire des sciences, les recherches de définitions et les études de méthodes interdisciplinaires $n$ 'ont certainement pas suffi à débrouiller un domaine encore très neuf. II ne faut pas nécessairement s'en plaindre. La multiplicité des approches est peut-être aussi le signe d'une vigoureuse vitalité et d'un avenir prometteur. Les sciences de l'information ont sans doute beaucoup de chemin à faire. L'essentiel était de montrer que dans l'état présent, il faut bien distinguer les professions de service documentaire et les sciences de l'information. La distance qui les sépare est encore grande puisque les premières, toujours pressées par des besoins urgents, utilisent et améliorent des outils techniques alors que les secondes, encore à l'état de recherches balbutiantes, s'attaquent à des phénomènes si fondamentaux que les métho-

41. Ian Mitroff et al, "Dialectical Inquiring Systems: a New Methodology for Information Science», Journal of the American Society for Information Science, vol. 23. no. 6 (November-December 1972), 365-378

42. F. Russo, "La pluridisciplinarité», 772

$43 \mathrm{C}$. West Churchman, The Design of Inquiring Systems, New York, Basic Books, 1971

44. Ces développements n'auraient pas été possibles sans l'optique résolument interdisciplinaire du programme en sciences de l'information de l'université de Pittsburgh. des d'investigation sont encore à trouver. Par conséquent, un savoir fondamental et directement exploitable est encore bien éloigné des professions documentaires. L'unité de la bibliothéconomie et de la documentation a été proposée comme le résultat d'un état de fait, d'ailleurs très récent. Nous avons voulu montrer qu'elle était plus: c'était une identité de service et de fonctions. Elle n'est pas encore le fruit d'une synthèse explicite ou d'un savoir scientifique constitué.

Il a cependant été possible de suggérer que les sciences de l'information sont des sciences humaines, par nature, interdisciplinaires. Ceci devrait permettre de préciser l'éventail des recherches à entreprendre si l'on veut mettre au service des professions documentaires une compréhension scientifique du phénomène information. 